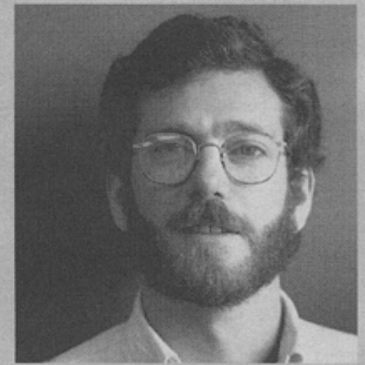

DANIEL J. VELLEMAN

Department of Mathematics and Computer Science Amherst College

Amherst, MA 01002 USA

Dan Velleman received his bachelor's degree from Dartmouth College in 1976 and his doctorate from the University of Wisconsin in 1980. He taught at the University of Texas and the University of Toronto before joining the faculty of Amherst College in 1983. He is interested in logic, philosophy of mathematics, and the foundations of quantum mechanics. $\mathrm{He}$ is the author of the book How to Prove It, and a coauthor, with Joe Konhauser and Stan Wagon, of the forthcoming problem collection Which Way Did The Bicycle Go? the consistency of ZF. His theorem is usually stated as saying, not that the continuum hypothesis is not provable in $\mathrm{ZF}$, but rather that if $\mathrm{ZF}$ is consistent then the continuum hypothesis is not provable.

To be sure, there is an important difference between Cohen's proof and Wiles's. The assumption that ZF is consistent is actually used in the reasoning of Cohen's proof, but Wiles's proof uses only ordinary mathematical reasoning and makes no reference to the consistency of ZF. It is only in thinking about whether or not Wiles's proof can be trusted that we are led to wonder about the consistency of $Z F$. For those who believe in the existence of the universe of all sets, and who believe that the $\mathrm{ZF}$ axioms are true statements about this universe, there is no reason to doubt the reliability of Wiles's proof. But for those who are skeptical about the existence of such a universe, the question remains: Should we be convinced by Wiles's proof that no counterexample to Fermat's Last Theorem will ever be found, or merely that if $\mathrm{ZF}$ is consistent then no counterexample will be found? ${ }^{13}$

I will not try to answer this question here. But I hope that by considering this question readers will be led to a greater appreciation of the significance of Hilbert's program, and the questions in the foundations of mathematics that remain unresolved to this day because of the failure of that program.

\footnotetext{
${ }^{13}$ Another way of thinking about this question is to consider formalizing the proof in different axiomatic systerns. As we have already observed, Wiles's proof is formalizable in ZF, but probably not in PA. Thus, Fermat's Last Theorem is provable in ZF, but it is still not known whether or not it is provable in PA. However, the statement "if $\mathrm{ZF}$ is consistent then Fermat's Last Theorem is true" can be encoded in the language of number theory (by Gödel numbering), and this encoded statement is provable in PA. The proof is simply the numerical encoding of our description of how a counterexample to Fermat's Last Theorem would lead to an inconsistency in ZF. Thus, the hypothesis that ZF is consistent is not needed for the proof of Fermat's Last Theorem if the proof is formalized in ZF, but it may be needed if the proof is formalized in PA. One's assessment of whether or not the hypothesis that ZF is consistent is needed for the proof of Fermat's Last Theorem might therefore depend on what axiom system one chooses to use.
}

\title{
Changing the Topology of the House Marion Cohen
}

You could identify various points like the corners or keyholes or pilot light.

You could identify various lines like fluorescent lights or woodwork.

You could identify various planes like the back door with the front or the floors with the ceilings.

You could identify the attic with the cellar or my room with my mother's.

You could take quotient spaces; two points could be equivalent if there exists a frictionless banister connecting them.

You could adjoin points, delete points; you could multiply this house by itself.

You could stuff up keyholes, clog the drainpipe.
You could even change the metric, or make it nonmetrizable; distances could mean nothing.

No matter what you do, however, this house will always be open.

Every point has its own sphere, and usually a large one.

Therefore this house will never be complete; in fact, inside this house, no sequence converges.

From The Weirdest is the Sphere, 2nd edition, 1990

\author{
Department of Mathematics \\ Temple University \\ Philadelphia, PA 19122 \\ USA
}

